\title{
6

\section{Comparison of Endoscopic Ultrasound Biopsy Needles for Endoscopic Ultrasound-Guided Liver Biopsy}

\author{
Armen Eskandari ${ }^{1}$, Patrick Koo ${ }^{1}$, Heejung Bang ${ }^{2}$, Dorina Gui ${ }^{3}$ and Shiro Urayama ${ }^{1}$ \\ ${ }^{1}$ Division of Gastroenterology and Hepatology, ${ }^{2}$ Department of Public Health Sciences, ${ }^{3}$ Department of Pathology and Laboratory Medicine, \\ University of California Davis, Davis, CA, USA
}

Background/Aims: To compare the performance of latest commercially available endoscopic ultrasound biopsy needles. Methods: Six latest commercially available needles were tested on a freshly harvested bovine liver; the tested needles included three $19 \mathrm{G}$, one $20 \mathrm{G}$, and two $22 \mathrm{G}$ needles. Five biopsies were performed per needle with $10 \mathrm{~mL}$ of wet suction. The primary outcome was the number of complete portal tracts (CPTs) per needle aspirate. The secondary outcomes were the mean specimen length and mean fragment length. Analysis of variance and Tukey's test were applied.

Results: All $19 \mathrm{G}$ needles and the $20 \mathrm{G}$ needle yielded similar mean CPTs and were superior to the SharkCore $22 \mathrm{G}$ needle $(p<0.001$ adjusted for multiplicity). There was no statistically significant difference in total specimen length among the three $19 \mathrm{G}$ needles and the $20 \mathrm{G}$ needle tested. The two $22 \mathrm{G}$ needles performed similarly with respect to the number of CPTs, mean fragment length, and mean specimen length (adjusted $p=0.07, p=0.59$, and $p=0.10$, respectively).

Conclusions: The specimen adequacy was similar among the 3 latest commercially available $19 \mathrm{G}$ needles. The endoscopist may choose a larger-bore needle based on availability without concerns of specimen adequacy. Further studies are needed to assess the ease of needle use in various anatomical locations and to confirm the optimal needle design. Clin Endosc 2019;52:347-352

Key Words: Endoscopic ultrasonography; Fine-needle biopsy; Liver

\section{INTRODUCTION}

Despite advances in image-based evaluation of parenchymal liver disease, a liver biopsy is still needed to determine the etiology or to grade the severity of the disease. The indication for liver biopsy becomes more compelling when a myriad of tests, including serology, imaging, and endoscopy (endoscopic retrograde cholangiopancreatography or endoscopic ultrasound [EUS]), have failed to yield a diagnosis. Traditionally, a percutaneous or transjugular approach is used for

Received: December 3, 2018 Revised: February 22, 2019

Accepted: February 24, 2019

Correspondence: Shiro Urayama

Division of Gastroenterology and Hepatology, University of California Davis, 1 Shields Ave, Davis, CA 95616, USA

Tel: +1-916-734-3751, Fax: +1-916-734-7608, E-mail: surayama@ucdavis.edu ORCID: https://orcid.org/0000-0001-6574-3161

(c) This is an Open Access article distributed under the terms of the Creative Commons Attribution Non-Commercial License (http://creativecommons.org/ licenses/by-nc/3.0) which permits unrestricted non-commercial use, distribution, and reproduction in any medium, provided the original work is properly cited. sampling of liver tissue. ${ }^{1,2}$ More recently, EUS has emerged as an ideal alternative for percutaneous liver biopsy in a subset of patients who would benefit from examination of the upper gastrointestinal tract, pancreas, and the biliary tree, and the surrounding vasculature in addition to visualization of the liver. EUS-guided liver biopsy has gained momentum, as studies have shown that it has comparable diagnostic yield to percutaneous biopsy. ${ }^{3-5}$ Newer needle designs have been introduced in parallel to this growing interest. However, few studies have compared the performance of different needle sizes and the needle designs. In this ex vivo study, we compared the performance of 6 latest commercially available needles, including the most recent $19 \mathrm{G}$ (3 needles), $20 \mathrm{G}$ (1 needle), and $22 \mathrm{G} \mathrm{(2}$ needles) models.

\section{MATERIALS AND METHODS}

\section{EUS fine-needle biopsy}

A freshly harvested bovine liver was procured from the 
University of California Davis Veterinary School Meat Laboratory (offered as a food item) and promptly used for liver biopsies. Six most recent models of commercially available needles were evaluated: Acquire 19 G/22 G (Boston Scientific, Marlborough, MA, USA), SharkCore 19 G/22 G (Medtronic, Minneapolis, MN, USA), EZ Shot 3 Plus 19 G (Olympus America, Center Valley, PA, USA), and EchoTip ProCore 20 G (Cook Medical, Bloomington, IN, USA). These needles vary in size and tip design, as shown in Fig. 1. The Acquire needle is a Franseen-type needle with 3 cutting tips. The SharkCore needle is a fork-tip-type needle with 2 parallel cutting tips. EZ Shot 3 Plus is a Menghini-type needle with a beveled end cutting edge; this needle is made of nitinol and is especially useful in lesions in difficult-to-reach locations, where there is excessive torque on the scope. Lastly, the ProCore needle has an end cutting beveled edge and a core trap near the tip. The advantages of the ProCore needle are flexibility because of its smaller gauge $(20 \mathrm{G})$ and the ability to provide a tissue core specimen owing to the side trap. Each needle was passed through a curvilinear Olympus echoendoscope (GF-UCT140; Olympus America) in a simulated flexed scope position to approximate the real-life tissue acquisition technique (Fig. 2). Real-time imaging was not performed (due to the large target). Five aspirates per needle were obtained with $10 \mathrm{~mL}$ of wet suction, resulting in a total of 30 core biopsy specimens. Each aspirate consisted of 5 actuations with $4-\mathrm{cm}$ needle advancement per actuation, performed by a single operator. Separate nonoverlapping locations from both lobes of the liver were chosen for each biopsy.

\section{Specimen preparation and data analysis}

The tissue cores were fixed in $10 \%$ formalin and processed according to the standard method. Paraffin wax-embedded

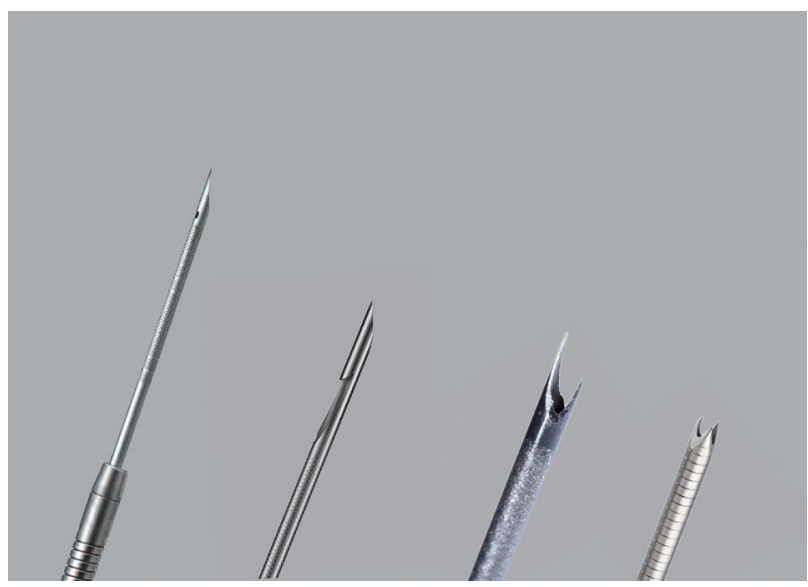

Fig. 1. Tip design of the needles used; from left to right: EZ Shot 3 Plus, EchoTip ProCore, SharkCore, and Acquire. sections were stained with hematoxylin and eosin (H\&E). The H\&E slides were scanned at $\times 20$ magnification using an Aperio AT2 scanner (Leica Microsystems GmBH, Wetzlar, Germany). All tissue measurements (total fragments and their lengths) were obtained using the Aperio Image Scope viewer (v12.3.2.8013)

The primary outcome was the number of portal tracts obtained per needle aspirate. A complete portal tract (CPT) was defined as containing all 3 portal structures (portal vein, hepatic artery, and bile duct). Two pathologists who were blinded to the needle type independently reviewed each sample and counted the number of CPTs. The secondary outcomes were the mean specimen fragment length per needle aspirate and the mean total specimen length. Comparisons between needle types for the 3 outcomes ( 1 primary outcome and 2 secondary outcomes, univariate analysis) were performed using analysis of variance (ANOVA) and Tukey's post-hoc analysis. All possible pairs of means were compared, and all $p$-values were adjusted for multiplicity. In the graphical representation of the difference in the measured outcomes, the letters $(A, B, C, D)$ indicate groupings where means are not statistically significantly different, with $5 \%$ alpha within the same letter.

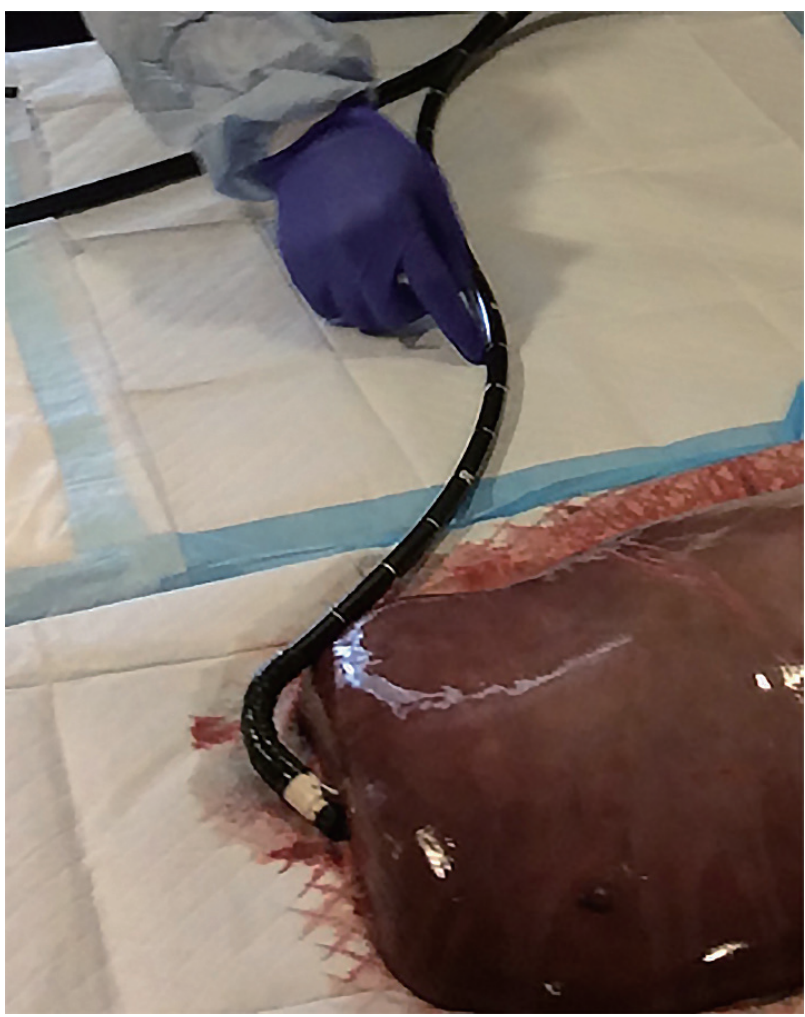

Fig. 2. Scope position during biopsy with an endoscopic ultrasound fine-needle aspiration needle. 
Table 1. Performance of Six Recent Commercially Endoscopic Ultrasound-Fine-Needle Biopsy Needles

\begin{tabular}{lccc}
\hline Needle type & Mean CPT $( \pm$ SD $)$ & Mean specimen length mm $( \pm$ SD $)$ & Mean fragment length mm $( \pm$ SD $)$ \\
\hline Shark core 19 G & $10.4 \pm 2.70$ & $51.50 \pm 4.61$ & $1.65 \pm 0.27$ \\
Acquire 19 G & $11.8 \pm 4.87$ & $71.30 \pm 20.23$ & $3.04 \pm 0.57$ \\
EZ Shot 3 Plus 19 G & $10.2 \pm 1.64$ & $71.77 \pm 12.86$ & $2.37 \pm 0.58$ \\
EchoTip ProCore 20 G & $7.2 \pm 1.48$ & $79.79 \pm 15.96$ & $2.02 \pm 0.46$ \\
Acquire 22 G & $6.4 \pm 2.79$ & $44.94 \pm 8.76$ & $1.33 \pm 0.29$ \\
SharkCore 22 G & $1.4 \pm 1.34$ & $20.89 \pm 11.56$ & $0.87 \pm 0.38$ \\
\hline
\end{tabular}

CPT, complete portal tract; SD, standard deviation.

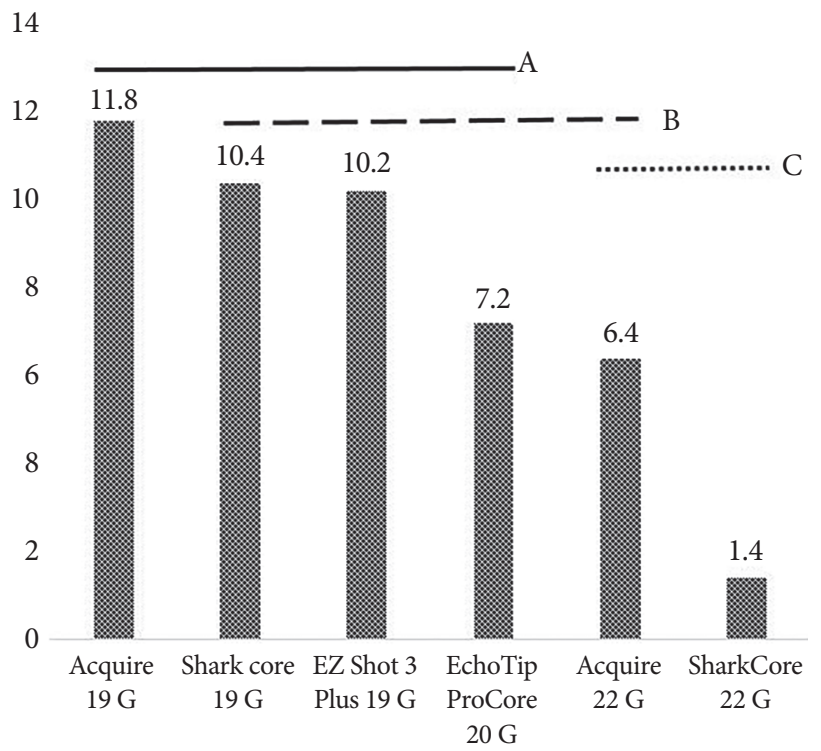

Fig. 3. Mean number of complete portal tracts per needle type. Variables that share the same letter line are not statistically different $(A, B, C)$.

\section{RESULTS}

The performance of the tested needles is summarized in Table 1 . The Acquire $19 \mathrm{G}$ needle yielded the highest mean number of CPTs (11.8 \pm 4.87$)$, whereas the SharkCore $22 \mathrm{G}$ yielded the lowest $(1.4 \pm 1.34, p<0.0001)$. However, as shown in Fig. 3, ANOVA demonstrated that all $19 \mathrm{G}$ and $20 \mathrm{G}$ needles yielded statistically similar mean CPTs. The yields of the larger-bore needles were superior to those of the $22 \mathrm{G}$ SharkCore needle ( $p<0.0001$ for Acquire 19 G, $p=0.0005$ for EZ Shot 3 Plus 19 G, $p=0.0004$ for SharkCore $19 \mathrm{G}, p=0.02$ for EchoTip ProCore 20 $\mathrm{G})$. For the mean total specimen length, the yield of EchoTip ProCore $20 \mathrm{G}$ was the longest and that of SharkCore $22 \mathrm{G}$ was the shortest $(79.8 \pm 15.9 \mathrm{~mm}$ vs. $20.9 \pm 11.5 \mathrm{~mm}, p<0.0001)$. There was no statistically significant difference in total specimen length among the three $19 \mathrm{G}$ needles and the $20 \mathrm{G}$ needle tested (Fig. 4).

The longest mean fragment length was obtained by Ac-

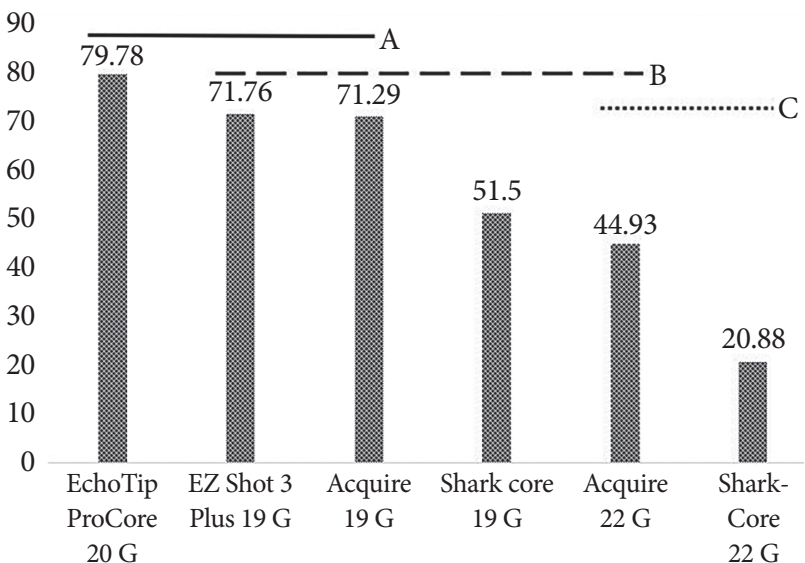

Fig. 4. Mean specimen length per needle type. Variables that share the same letter line are not statistically different $(A, B, C)$.

quire $19 \mathrm{G}(3.04 \pm 0.27 \mathrm{~mm})$, whereas the shortest fragment was obtained by SharkCore $22 \mathrm{G}(0.87 \pm 0.380 \mathrm{~mm}, p<0.0001)$. The Acquire $19 \mathrm{G}$ needle yielded statistically longer tissue fragments than all the other needle types tested, except for the EZ Shot 3 Plus 19 G needle (Fig. 5). There was no statistically significant difference between the two $22 \mathrm{G}$ needles examined in terms of the number of CPTs, mean fragment length, and mean total specimen length ( $p=0.07, p=0.59$, and $p=0.10$, respectively).

\section{DISCUSSION}

Liver biopsy is commonly the final step in the evaluation of liver disease and is regularly used to objectively measure the degree of liver fibrosis. Recently, EUS has emerged as a useful tool for obtaining liver biopsy specimens, as it provides real-time guidance for the target, including identification of focal lesions as well as of the presence of prominent portal hypertensive vasculature patterns. ${ }^{6,7}$ Among several factors that determine the usefulness of a liver biopsy specimen for diagnosis and staging, 2 main variables have been suggested, 


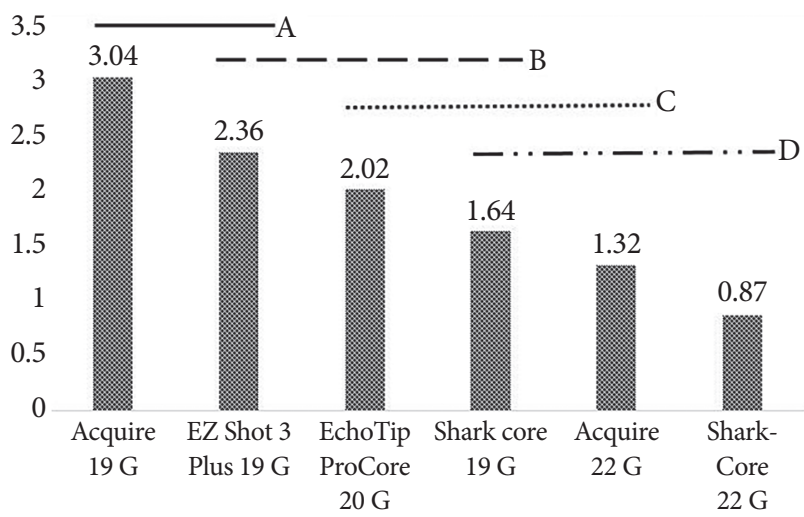

Fig. 5. Mean fragment length per needle type. Variables that share the same letter line are not statistically different $(A, B, C, D)$.

namely specimen length and number of CPTs. It is generally agreed that the specimen should be at least $15 \mathrm{~mm}$ in length and contain 6-8 CPTs. ${ }^{8}$ In prospective studies on EUS-guided needle biopsy using fine-needle aspiration (FNA) or fine-needle biopsy (FNB) needles, specimen adequacy criteria of 6 or more CPTs and $15 \mathrm{~mm}$ specimen length were reached in $>90 \%$ of the cases. ${ }^{9,10}$ As interest in EUS-guided liver biopsy has been growing, newer needle designs have become available to improve the yield of this procedure.

In this ex vivo study, we compared the yields among more comprehensive, recently available $19 \mathrm{G}(3), 20 \mathrm{G}(1)$, and 22 $\mathrm{G}$ (2) needles with various needle tip geometries, with the number of CPTs as the primary outcome. We found no significant difference among the three $19 \mathrm{G}$ needles and the single $20 \mathrm{G}$ needle for the primary outcome. The performance of some needle designs in liver biopsy has been compared in 2 previous ex vivo studies. ${ }^{11,12}$ Lee et al. compared four $19 \mathrm{G}$ FNA needles (Cook EchoTip ProCore, Olympus EZ Shot 2, Boston Scientific Expect Slimline, and Medtronics SharkCore) and one 18 G Tru-Cut percutaneous needle (TruCore; Argon Medical Devices, Frisco, TX, USA) in 2 normal human cadaveric livers. ${ }^{12} \mathrm{~A}$ total of 12 biopsy specimens were taken using each needle, with 3,6 , and 9 actuations in each pass. ${ }^{12}$ In the study, the SharkCore $19 \mathrm{G}$ needle yielded significantly higher number of CPTs (mean 8.83) than the other $19 \mathrm{G}$ needles. The 18 G Tru-Cut percutaneous needle yielded 7 CPTs $(p>0.05)$. In comparison, our current study also incorporated the newly employed Franseen-type (Acquire) and Menghini-type (EZ Shot 3 Plus) EUS-FNB needle tip designs in addition to the fork-tip needle (SharkCore). The utilization of the needles with their redesigned tip geometry with improved yield is supported by a recent report on the Franseen vs. fork-tip types of needle in sampling of pancreatic mass lesions. ${ }^{13}$ Other potential causes of the variation in results may include the different biopsy techniques and the use of fresh liver specimen

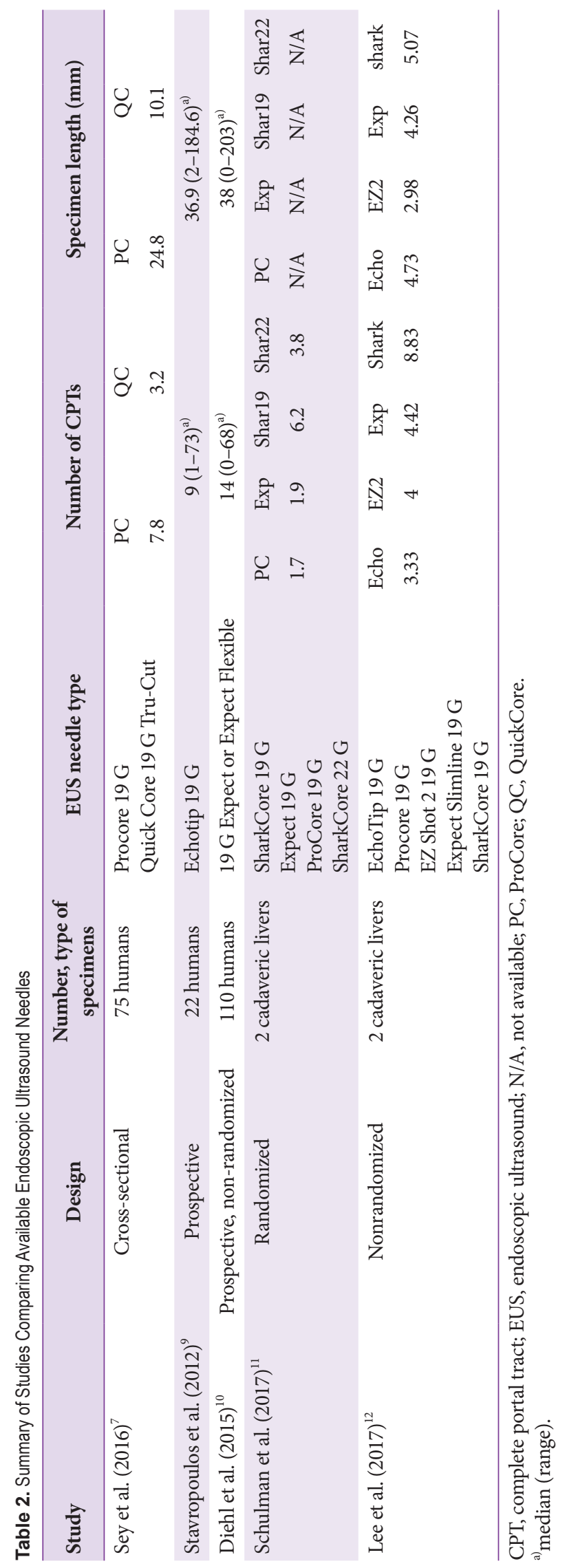


in our study, in contrast to the use of a cadaveric liver with potentially different tissue or cellular cohesiveness.

The significance reached in the study by Lee et al. may also be derived from the relatively low number of CPTs obtained by the other (Expect, EchoTip, and EZ shot) 19 G needles, which have the traditional needle-tip design, in comparison to other previous studies. ${ }^{12}$ For instance, Diehl et al. used Expect or Expect Flexible needles in 110 human subjects, and reported a median CPT of 14 compared to the reported mean of 4.42 by Lee et al ${ }^{10,12}$ Furthermore, 105 of the 110 patients in the study had $>6$ CPTs. ${ }^{10}$ As for the EchoTip needle, whereas Lee et al. reported a mean CPT of 3.3, Stavropoulos et al. reported a median CPT of 9 with $91 \%$ of patients having $>6$ CPTs. ${ }^{9,12} \mathrm{As}$ for the SharkCore $19 \mathrm{G}$ needle, we reported a mean CPT of 10.4, which is similar to the $8.8 \mathrm{CPTs}$ reported by Stavropoulos et al. ${ }^{9}$

In another ex vivo study on 2 human cadaveric livers by Schulman et al., three $19 \mathrm{G}$ needle types (SharkCore FNB, Expect FNA [Boston Scientific, Natick, MA, USA], and ProCore FNB [EchoTip HD ProCore]) and one $22 \mathrm{G}$ (SharkCore FNB) needle were compared with 2 percutaneous $18 \mathrm{G}$ (QuickCore [Cook Medical] and Coaxial Temno [Care-Fusion, McGaw Park, IL, USA]) needles for the primary outcome of number of CPTs. ${ }^{11}$ In this study, in which a total of 288 biopsy specimens were taken (48 per needle type), the 19 G SharkCore needle outperformed all the other needle types with a mean CPT of 6.2. Additionally, the 22 G SharkCore FNB needle yielded significantly more portal tracts than the Expect FNA (3.8 vs. $1.9, p=0.004$ ), ProCore FNB (3.8 vs. $1.7, p=0.012$ ), and percutaneous $18 \mathrm{G}(3.8$ vs. $2.5, p=0.05)$ needles. ${ }^{11}$ It is worth mentioning that although the number of CPTs in our work is closer to that reported in previous human studies, the CPTs obtained by the $19 \mathrm{G}$ needles in both the studies by Lee et al. and Schulman et al. were lower than the $19 \mathrm{G}$ needle yields in previous reports on humans. ${ }^{11,12}$ The mechanistic explanation for this discrepancy is lacking but this difference may also be related to the use of cadaveric liver and/or differences in the aspiration technique. Table 2 shows a comparison of the current study with previous studies on EUS-guided liver biopsy with non-Tru-Cut needles.

Our study has limitations that merit discussion. We paid attention to using a fresh liver specimen and to the scope positioning to mimic real-life tissue sampling as much as possible. Although this effort enabled obtaining a range of CPT counts close to those obtained in previous in vivo human studies, there may be differences in real-life tissue sampling arising from anatomical and technical variations for each patient and operator. In addition, the use of a bovine specimen in our study might raise concerns; however, it seems reasonable to consider that the performance can be translated to human liver specimens as the bovine liver shares the overall anatomical and physical properties of the human liver. ${ }^{14}$ Finally, as we experimented on an ex vivo liver model, adverse events could not be assessed.

In conclusion, the literature is scarce with respect to EUS-guided liver biopsy techniques that yield the optimal specimen for pathological analysis. The available studies considerably vary in needle design and biopsy technique. Our current study provides an initial, but revealing, data demonstrating comparable outcomes with the use of multiple newly designed $19 \mathrm{G}$ needles for liver biopsy. This finding is clinically relevant because it suggests that the endoscopist can choose a needle based on his or her comfort and experience and institutional availability without concerns over diagnostic performance. Further studies may be needed to assess the ease of needle use in various anatomical locations and for confirming the optimal needle design in a larger sample.

\section{Conflicts of Interest}

The authors have no financial conflicts of interest.

\section{Acknowledgements}

We would like to thank Seuneh Petrosian for her assistance with the design of the figures.

The project described was supported by the National Center for Advancing Translational Sciences, National Institutes of Health, through grant number UL1 TR001860. The content is solely the responsibility of the authors and does not necessarily represent the official views of the NIH.

\section{Author Contributions}

\section{Conceptualization: Shiro Urayama}

Data curation: Armen Eskandari, Patrick Koo, Dorina Gui, SU

Formal analysis: AE, Heejung Bang, $\mathrm{SU}$

Methodology: PK, DG

Supervision: $\mathrm{SU}$

Writing-original draft: $\mathrm{AE}$

Writing-review\&editing: $\mathrm{AE}, \mathrm{SU}$

\section{REFERENCES}

1. Kalambokis G, Manousou P, Vibhakorn S, et al. Transjugular liver biopsy--indications, adequacy, quality of specimens, and complications--a systematic review. J Hepatol 2007;47:284-294.

2. Rockey DC, Caldwell SH, Goodman ZD, Nelson RC, Smith AD. Liver biopsy. Hepatology 2009;49:1017-1044.

3. Pineda JJ, Diehl DL, Miao CL, et al. EUS-guided liver biopsy provides diagnostic samples comparable with those via the percutaneous or transjugular route. Gastrointest Endosc 2016;83:360-365.

4. Gleeson FC, Clayton AC, Zhang L, et al. Adequacy of endoscopic ultrasound core needle biopsy specimen of nonmalignant hepatic parenchymal disease. Clin Gastroenterol Hepatol 2008;6:1437-1440.

5. Gor N, Salem SB, Jakate S, Patel R, Shah N, Patil A. Histological adequacy of EUS-guided liver biopsy when using a 19-gauge non-Tru-cut FNA needle. Gastrointest Endosc 2014;79:170-172.

6. Dewitt J, McGreevy K, Cummings O, et al. Initial experience with 
EUS-guided tru-cut biopsy of benign liver disease. Gastrointest Endosc 2009;69(3 Pt 1):535-542.

7. Sey MS, Al-Haddad M, Imperiale TF, McGreevy K, Lin J, DeWitt JM. EUS-guided liver biopsy for parenchymal disease: a comparison of diagnostic yield between two core biopsy needles. Gastrointest Endosc 2016;83:347-352.

8. Bravo AA, Sheth SG, Chopra S. Liver biopsy. N Engl J Med 2001; 344:495-500

9. Stavropoulos SN, Im GY, Jlayer Z, et al. High yield of same-session EUS-guided liver biopsy by 19-gauge FNA needle in patients undergoing EUS to exclude biliary obstruction. Gastrointest Endosc 2012;75:310318.

10. Diehl DL, Johal AS, Khara HS, et al. Endoscopic ultrasound-guided liver biopsy: a multicenter experience. Endosc Int Open 2015;3:E210-E215.
11. Schulman AR, Thompson CC, Odze R, Chan WW, Ryou M. Optimizing EUS-guided liver biopsy sampling: comprehensive assessment of needle types and tissue acquisition techniques. Gastrointest Endosc 2017;85:419-426.

12. Lee WJ, Uradomo LT, Zhang Y, Twaddell W, Darwin P. Comparison of the diagnostic yield of EUS needles for liver biopsy: ex vivo study. Diagn Ther Endosc 2017;2017:1497831.

13. Bang JY, Hebert-Magee S, Navaneethan U, Hasan MK, Hawes R, Varadarajulu S. Randomized trial comparing the Franseen and fork-tip needles for EUS-guided fine-needle biopsy sampling of solid pancreatic mass lesions. Gastrointest Endosc 2018;87:1432-1438.

14. Kemper AR, Santago AC, Stitzel JD, Sparks JL, Duma SM. Biomechanical response of human liver in tensile loading. Ann Adv Automot Med 2010;54:15-26. 